\title{
Das Recht auf den eigenen Tod - Patientenverfügungen als Autonomie für die Stunde des Todes?
}

\section{Patientenrechte und Selbstbestimmung}

Das Selbstbestimmungsrecht eines Menschen kommt zum Ausdruck durch eigenverantwortliche Entscheidungen. So ist es ein wesentliches Anliegen jedes Menschen, Einfluss auf eine ärztliche Behandlung zu nehmen und ärztlichem Handeln nicht ausgeliefert zu sein. Dies wird offensichtlich durch die eigene Wahl des Arztes oder Krankenhauses.

Ein entscheidungsfähiger Mensch trifft nach dem Prinzip des »informed consent « (Einwilligung nach Aufklärung) seine Entscheidungen selbst. Dazu gehört auch die Möglichkeit zu Entscheidungen, die andere Menschen nicht als vernünftig ansehen. Der Essener Medizinethiker Quante betont die Möglichkeit zur Meinungsbildung über zukünftige Situationen, wenn er schreibt: »Sein Leben als Person zu führen bedeutet, sich wertend mit der eigenen Zukunft zu identifizieren. « ${ }^{1}$ Das Recht auf Selbstbestimmung wird als eines der wichtigsten Rechte angesehen. Bemerkenswert ist, dass das Prinzip der Selbstbestimmung oder Autonomie keine homogene Theorie darstellt, sondern aus verschiedenen Moralsystemen ableitbar ist und entsprechend auch teils kontrovers interpretiert wird. Respekt vor der Autonomie des Patienten gilt - unabhängig von der jeweiligen Begründung - als ein zentrales ethisches Prinzip. Der amerikanische Sozialphilosoph Feinberg unterscheidet zwischen vier zentralen Bedeutungen von Autonomie:

1. Autonomie als persönliche Fähigkeit (capacity)

2. Autonomie als situative Disposition (actual condition)

3. Autonomie als Charakterideal (ideal of character)

4. Autonomie als moralisches Recht (sovereign authority). ${ }^{2}$

In den ersten beiden Bedeutungen ist Autonomie ein deskriptiver Begriff, der eine Fähigkeit oder eine Situation beschreibt. In den beiden anderen Bedeutungen handelt es sich um einen normativ-wertenden Begriff - wenn auch in verschiedenen Hinsichten: Autonomie als moralisches Recht ist etwas, das man einfordern kann, Autonomie als Charakterideal etwas, das man lediglich empfehlen kann. Autonomie als moralisches Recht impliziert, dass andere eine entsprechende Pflicht zu seiner Wahrung und Achtung haben, während Autonomie als Charaktereigenschaft als solches zu nichts verpflichtet. Andererseits lässt sich auch zwischen den ersten drei Bedeutungen und der vierten eine kategoriale Grenze ziehen: In den drei ersten Bedeutungen bezeichnet Autonomie eine (faktische oder erwünschte) Fähigkeit, in der vierten einen Anspruch. Autonomie als Rechtsanspruch bedeutet nicht, dass jemand selbstbestimmt ist, son-

1 Quante, M. (2002): Personales Leben und menschlicher Tod, S. 286.

2 Feinberg, J. (1986): Harm to self, S. 28. 
dern dass er ein Recht auf Selbstbestimmung hat, dass bestimmte Dinge nicht gegen oder ohne seine Zustimmung mit ihm geschehen sollen.

Im Arzt-Patient-Verhältnis spielen alle vier Bedeutungen des Autonomiebegriffs eine zentrale Rolle: Autonomie im Fähigkeitssinn (Bedeutung 1 und 2) im Kontext der Frage der Einwilligungsfähigkeit, Autonomie als Ideal (Bedeutung 3) im Sinne einer regulativen Idee der Arzt-Patient-Beziehung, Autonomie als Rechtsanspruch (des Patienten; Bedeutung 4) als normative Grundlage der Arzt-Patient-Beziehung in allen Fällen einer medizinischen Intervention, die nicht den Charakter einer Notfall- und Krisenintervention haben.

Die juristische Verankerung des Selbstbestimmungsrechtes in Zusammenhang mit dem Prinzip der Aufklärung findet sich in dem verfassungsrechtlich verbürgten Recht auf körperliche Unversehrtheit (Art. 2 II 1 GG), dem eng damit verknüpften allgemeinen Persönlichkeitsrecht (Art. 2 I 1 GG) sowie der durch Art. 1 I 1 GG verbürgten Menschenwürde. Aufgrund dieser Rechte ist es jedem entscheidungsfähigen Erwachsenen erlaubt, selbst über seinen Körper zu entscheiden; d.h. eine medizinische Behandlung zu autorisieren oder auch zu verweigern, und sei die Verweigerung aus der Sicht anderer noch so unvernünftig oder sogar lebensbedrohlich. Der Bundesgerichtshof (BGH) führt aus: »Denn ein selbst lebensgefährlich Kranker kann triftige Gründe haben, eine Operation abzulehnen, auch wenn er durch sie und nur durch sie von seinem Leiden befreit werden könnte. ${ }^{3}$

Zur Einwilligungsfähigkeit stellte der BGH 1953 fest, dass der Einwilligende die »nötige Urteilskraft und Gemütsruhe « besitzen muss, »um die Tragweite seiner Erklärung für das Für und Wider verständig gegeneinander abzuwägen « ${ }^{4} .1969$ änderte der BGH die Umschreibung so, dass der Einwilligende »die ausreichende Urteilsfähigkeit über Wesen, Bedeutung und Tragweite der gegen sie gerichteten Handlung ${ }^{5}{ }^{5}$ besitzen müsse. Einwilligungsfähigkeit wird also mit Einsichtsfähigkeit gleichgesetzt. Diese setzt voraus, dass der Patient auf Grundlage eines individuellen, seinem Auffassungsvermögen entsprechenden Aufklärungsgesprächs Art, Umfang und Bedeutung des Eingriffs $\mathrm{zu}$ verstehen vermochte und eine Nutzen-Risikoabwägung vorgenommen hat. Bei der Feststellung der Einwilligungsfähigkeit stellt sich in der Praxis mitunter das Problem, dass die Beurteilung der Entscheidungsfähigkeit eine Ja/Nein-Entscheidung erfordert, wobei die Voraussetzungen für diese Fähigkeiten beim Patienten graduell vorliegen. Es geht also um eine Schwellenentscheidung, die von unterschiedlichen Ärzten unterschiedlich beurteilt werden kann. Das Recht auf Selbstbestimmung und das damit verbundene Vetorecht gegenüber medizinischen Maßnahmen gelten grundsätzlich aber auch für Situationen, in denen der Patient nicht mehr in der Lage ist, seinen Willen aktuell zu äußern. Voraussetzung ist, dass der Patient im Vorfeld dieser Situation seinen Willen vorsorglich - z.B. in Form einer Patientenverfügung bekundet hat.

3 BGH NJW 1957, S. 268.

4 BGHSt 4, 88, [90].

5 BGHSt 23, 1, [4]. 


\section{Entwicklung Vorsorgender Verfügungen}

Bei vielen Menschen ist das Bedürfnis vorhanden, Entscheidungen über die eigene Behandlung zu beeinflussen, selbst dann wenn die direkte Entscheidungsmöglichkeit nicht mehr vorhanden ist. Dieser Wunsch rührt auch von der Befürchtung, vom entscheidungsfähigen Subjekt zum Objekt einer Behandlung zu werden, die vom Patienten nicht gewünscht wird. Dieses Motiv für eine Vorsorgende Verfügung hat stärker abwehrenden Charakter, wobei auch oft gestalterische Elemente vorhanden sind.

Technische Möglichkeiten einer spezialisierten Intensivmedizin rufen manchmal Befürchtungen hervor, von einer hochtechnisierten, seelenlosen Apparatemedizin abhängig zu sein, bei der der Mensch nur noch an »Schläuchen « hängt und seiner Lebensqualität beraubt ist.

Der Erfurter Moraltheologe Römelt sieht in der »Retardierung der Alterungs- und Sterbeprozesse« durch »Eingriffschancen der modernen Medizin« eine deutlicher und ausführlicher ins Bewusstsein kommende »Unausweichlichkeit und Härte des Sterbens $\ll{ }^{6}$

Aus dem verständlichen Wunsch Einfluss auf eine zukünftige, medizinische Behandlung zu nehmen, insbesondere Maßnahmen, wie maschinelle Beatmung und Reanimation, in Extremfällen auch abzulehnen, entstanden Patientenverfügungen. Als Vorausverfügungen (engl. Advance Directives) oder Patientenverfügungen bezeichnet, sollen sie Richtlinien für zukünftige Situationen festlegen, in denen ein Patient selbst nicht mehr medizinische Behandlung autorisieren oder ablehnen kann. Man kann sie daher als Kommunikationsbrücken verstehen, die Zustände erreichen sollen, in denen eine direkte Kommunikation nicht mehr möglich ist. Die zugrundeliegende Idee der Patientenverfügung erscheint sehr attraktiv, da eine Reihe der schwierigen Entscheidungen, etwa bei komatösen Patienten, zumindest theoretisch entsprechend den persönlichen Wünschen der Patienten getroffen werden könnten. Bedeutung erlangt in diesem Zusammenhang die schriftliche Form, die 1969 von Kutner erstmals erwähnt und »living will« genannt wurde ${ }^{7}$. Die deutsche Diskussion erhielt wesentliche Impulse durch den Kölner Konkursrichter Uhlenbruck, der als Analogie der Kommunikation des Arztes mit Kollegen und dem Patienten durch einen Arztbrief nun den Patientenbrief vorstellte ${ }^{8}$. Es werden in der Diskussion weitere Begriffe wie Patientenschutzbrief ${ }^{9}$, $»$ Euthanasie-Testament $\ll^{10}$, Patientenanwaltschaft, Patientenanwaltsverfügung ${ }^{11}$, »Patientenerklärung « ${ }^{12}$, $\gg$ Kranken- und Schwerverletztenverfü-

6 Römelt, J. (2004): Menschenwürdiges Sterben, Herder Korrespondenz, S. 58.

7 Kutner, L. (1969): Due process of euthanasia: the living will, a proposal, Indiana Law Journal, S. 539-554.

8 Uhlenbruck, W., Der Patientenbrief - die privatautonome Gestaltung des Rechts auf einen menschenwürdigen Tod, NJW 1978, S. 566-570.

9 Deutsche Gesellschaft für humanes Sterben (DGHS) (1999): Patientenschutzmappe.

10 Uhlenbruck, $W$., Der Patientenbrief - die privatautonome Gestaltung des Rechts auf einen menschenwürdigen Tod, NJW 1978, S. 566.

11 DGHS (1999): Patientenschutzmappe.

12 Arbeitskreis »Arzt und Seelsorger « bei der Evangelischen Akademie Iserlohn (1999): Für sich selber sorgen bis zum Ende. Leitfaden zum Erstellen einer persönlichen Patientenerklärung. 
gung ${ }^{13}$, »Patientenletztverfügung ${ }^{14}{ }^{4}, \quad$ Patientenvorausverfügung ${ }^{15}$, Lebenstestament $^{16}$, »Willenserklärung ... für lebensbedrohliche Situationen ${ }^{17}{ }^{17}$ Vorabverfügung, Vorausverfügung, »Vorab-Erklärung ${ }^{18}{ }^{18}$, Vorsorgliche Verfügung verwendet.

In der Diskussion und im allgemeinen Sprachgebrauch hat sich der Begriff der Patientenverfügung durchgesetzt. Der Begriff Patienten«testament« ist wegen der sprachlichen Nähe zu einem unwiderruflichen Dokument für die Zeit nach dem Tode nicht zu empfehlen, denn eine Patientenverfügung ist für die Zeit des Lebens mit eingeschränkter Kommunikationsfähigkeit gedacht.

Wenn ein Patient sich selbst nicht mehr äußern kann, fehlt den behandelnden Ärzten und dem Pflegepersonal ein direkter Ansprechpartner. Für diese Fälle besteht die Möglichkeit der vorsorglichen Willensbekundung in Form einer Patientenverfügung sowie der Bevollmächtigung einer Person, die stellvertretend für den nicht mehr einwilligungsfähigen Patienten Entscheidungen trifft, mittels einer Vorsorgevollmacht. Durch eine Betreuungsverfügung kann Einfluss auf die Auswahl des Betreuers genommen werden ( $\$ 1897$ IV 3 BGB).

Die beiden Instrumente der Vorsorgevollmacht und Betreuungsverfügung beziehen sich auf die Übertragung der Entscheidungsbefugnis. Konkrete Wünsche zur medizinischen Versorgung können vorab durch eine mündliche oder schriftliche Patientenverfügung festgelegt werden. Mit einer Patientenverfügung kann ein Patient bestimmen, wie und in welchem Umfang er in bestimmten Krankheitssituationen behandelt werden möchte. Die Patientenverfügung ist dabei nicht zwangsläufig Ausdruck einer vorgefassten Ablehnung bestimmter Therapien.

Als anerkanntes Prinzip gilt in der Medizin und Medizinethik das Prinzip der Einwilligung nach Aufklärung. Ein Patient muss vor einem Eingriff über die relevanten Fakten und Vor- und Nachteile aufgeklärt werden. Die dann folgende Entscheidung eines einwilligungsfähigen Patienten ist bindend, so unvernünftig sie erscheinen mag.

Die Vielfalt der technischen Möglichkeiten in der Medizin führt nicht zu einer einhelligen Meinung aller Patienten zu bestimmten Eingriffen. Die Patientenverfügung eignet sich in besonderer Weise für die Kommunikation moralisch Fremder, die sich nicht kennen. Die Bundesärztekammer fordert eine individuelle, auf die Wünsche des

13 Haarhaus, F., Neues zu Kranken- und Schwerverletztenverfügung, Die Schwester/Der Pfleger 1999, S. 782-785 \& S. 877-880.

14 Hospizarbeit im Evangelischen Johanneswerk (Hrsg.) (1999): Patientenletztverfügung. Fragen und Antworten.

15 Höfling, W./Demel, M., Zur Forschung an Nichteinwilligungsfähigen, MedR 1999, S. 546.

16 Europarat, Parlamentarische Versammlung, Ausschuss für Sozialordnung, Gesundheit und Familie (1999): Entwurf einer Empfehlung betr. den Schutz der Menschenrechte und der Würde todkranker und sterbender Menschen vom 21.05.1999, Dokument Nr. 8421, Übersetzung des Bundestages, Punkt 9, 4.

17 Internationale Gesellschaft für Sterbebegleitung und Lebensbeistand e.V. (IGSL) (1999): Willenserklärung und Betreuungsverfügung für lebensbedrohliche Situationen; dazu IGSL (2000): Vorbereitungsmaterial für Aussteller von Vorausverfügungen zu lebensverlängernden Maßnahmen.

18 Uhlenbruck, $W$., Die Altersvorsorge-Vollmacht als Alternative zum Patiententestament und zur Betreuungsverfügung, NJW 1996, S. 1583. 
Patienten eingehende Behandlung. Dazu bietet eine Patientenverfügung die nötige Grundlage.

Nach dem Ansatz der Befähigung zur Gesundheitsmündigkeit müssen entscheidungsrelevante Informationen zur Verfügung stehen, die mögliche Entscheidungskonflikte der medizinischen Behandlung transparent machen. Dies kann durch Fallvignetten erfolgen, auf Grund derer Interessierte mit Therapiemöglichkeiten und Entscheidungsoptionen vertraut gemacht werden. Diese Beratung und Information ${ }^{19}$ muss wertneutral erfolgen und es darf kein Zwang auf Ratsuchende ausgeübt werden. Eine Patientenverfügung stellt somit eine individuelle Entscheidung eines Menschen dar und ist kein Werturteil einer Gesellschaft über einen Bewusstseinszustand. Auch eine Erodierung der Rechte von Patienten im Chronischen Apallischen Syndrom (PVS; Wachkoma) zu befürchten, ist abwegig, da in Deutschland der Grundsatz des Lebensschutzes gilt, von dem ich bei abweichender Meinung durch eine direkte Meinungsäußerung oder eine Patientenverfügung abweichen kann (aber nicht muss). Dies setzt eine bewusste Entscheidung voraus.

Es besteht ein wesentlicher Unterschied zwischen der negativen Freiheit in Ruhe gelassen zu werden, - also eine Therapie ablehnen zu können - und der positiven Freiheit auf bestimmte Behandlungsoptionen. Die Ablehnung und das Fordern von medizinischer Behandlung scheinen daher unterschiedliche moralische Stringenz haben. Manche sprechen auch von Defensivrechten im Gegensatz zu Teilhaberechten. Eine weitere wesentliche Begrenzung des Selbstbestimmungsrechtes besteht in der Regel darin, dass es an bestimmte Bedingungen geknüpft ist. Der im angloamerikanischen Common Law besonders gerne zitierte und berühmte Spruch des Richters Cardozo beschränkte das Selbstbestimmungsrecht auf zurechungsfähige Erwachsene: "Every human being of adult years and sound mind has a right to determine what shall be done with his own body." ${ }^{20}$

In der Entscheidung des Bundesgerichtshofs vom 13.09.1994 werden Kriterien für die erlaubte sog. passive Sterbehilfe genannt und folgende Kriterien zur Ermittlung des mutmaßlichen Willens benannt: »frühere mündliche oder schriftliche Äußerungen des Patienten, seine religiöse Überzeugung, seine sonstigen persönlichen Wertvorstellungen, seine altersbedingte Lebenserwartung oder das Erleiden von Schmerzen $\ll^{21}$. Dabei festigt der Bundesgerichtshof den Grundsatz des Lebensschutzes. Sollte ein individueller mutmaßlicher Wille nicht erkennbar und ermittelbar sein, so muss der Schutz menschlichen Lebens Vorrang haben: »In dubio pro vita«.

Im Prinzip der Doppelwirkung kommt die Überlegung des Bundesgerichtshofs bei der Entscheidung zur sog. indirekten Sterbehilfe zum Ausdruck und trotz möglicherweise lebensverkürzenden Nebenwirkungen ist für den Bundesgerichtshof 1996 der Tod in Würde und Schmerzfreiheit ein »höherwertiges Rechtsgut als die Aussicht,

19 Vgl. auch Entwurf eines 3. Gesetzes zur Änderung des Betreuungsrechts vom 1.11.2004, S. 8; online unter http://www.aem-online.de; siehe dazu die Pressemeldung vom 5.11.2004, abrufbar unter: http://www.bmj.bund.de/media/archive/791.pdf.

20 Schloendorff v., Society of New York Hospital (1914) 211 NY 125 at 126.

21 BGHSt 40, $257 \mathrm{ff}$. 
unter schwersten, insbesondere sog. Vernichtungsschmerzen noch kurze Zeit länger leben zu müssen $\aleph^{22}$.

Im zeitlichen Ablauf spielten Patientenverfügungen in den 80er Jahren des vergangenen Jahrhunderts vor allem eine Rolle in der Diskussion um die Frage, ob aktive Sterbehilfe in Einzelfällen ethisch vertretbar sei. Die Deutsche Gesellschaft für Humanes Sterben (DGHS) stellte 1981 nach eigenen Angaben erstmalig in Deutschland eine Verfügung mit der Bezeichnung Patientenverfügung ${ }^{23}$ vor. Die DGHS veröffentlichte einen Patientenschutzbrief und eine nur für Mitglieder erhältliche Freitodverfügung. Spektakuläre Medienauftritte bestimmter Ärzte und Organisationen sollten in der Öffentlichkeit für eine »kontrollierte ärztliche Erlösungstodhilfe aus Barmherzigkeit« werben. Als Beispiel für dieses Anliegen darf das Verfügungsdokument von Hackethal gelten.

Seitdem fällt ein Schatten auf alle Patientenverfügungen. Kritiker machen ihnen bis heute zum Vorwurf, Menschen dazu zu verführen, höchst subjektive, damit moralisch anfechtbare und für Schwankungen anfällige, Urteile über Maßstäbe zu fällen, die Vorstellungen von Lebensqualität betreffen. Mancher wittert in der Patientenverfügung eine regelrechte »Autonomiefalle« und verwirft sie als Einwilligung in eine assistierte Tötung unter dem Tarnmantel freier Selbstbestimmung. Ein Echo findet diese ablehnende Haltung dort, wo Patientenverfügungen zwar angeboten werden, ihre Reichweite aber - wie z.B. bei der Christlichen Patientenverfügung der christlichen Kirchen 1999 - zugleich auf die Situation der Sterbephase begrenzt wird. Dabei sind Patientenverfügungen nicht Ausdruck des Wunsches nach Sterbe»hilfe«, sondern vor allem nach ärztlicher Sterbebegleitung.

In den 90er Jahren des vergangenen Jahrhunderts wuchs das Interesse in der Bevölkerung an einer Patientenverfügung kontinuierlich. Im Zusammenhang mit Gerichtsentscheidungen zum Behandlungsabbruch, z.B. bei der Einstellung der künstlichen Ernährung, wurde die Bedeutung des Patientenwunsches in Form einer Patientenverfügung hervorgehoben. Hospizbewegung, einige Interessenverbände wie Ärztekammern, Anwaltvereine, Kommunalbehörden, Seniorenverbände und Verbraucherzentralen, schließlich auch die Kirchen und diakonische Einrichtungen nahmen sich des Themas an und entwickelten eigene Verfügungsmodelle. Zeitgleich wurden weltweit in wissenschaftlichen Fachkreisen Studien zur ethischen und rechtlichen Beurteilung von Vorausverfügungen erstellt, sowie Fragen klinischer Umsetzbarkeit erörtert. Umfragen bei Ärzten und Patienten wurden durchgeführt, Modelle in Krankenhäusern und Pflegeeinrichtungen erprobt. Schließlich wurden die oben genannten Instrumente der Vorsorgevollmacht bzw. Betreuungsverfügung immer wichtiger. Der Gesetzgeber überarbeitete zum 01.01.1999 das Betreuungsrecht und stärkte damit die Bedeutung der Vorsorgevollmacht im Bereich der Gesundheitsangelegenheiten. Sie wird heute mit fast allen gegenwärtig vorhandenen Verfügungen kombiniert.

22 BGHSt 42, 301 (305).

23 Der ehemalige Geschäftsführer der DGHS Atrott reklamiert den Begriff der Patientenverfügung 1981 erstmals eingeführt und geprägt zu haben. 


\section{Patientenverfügungsmuster und Formulierungsvorschläge}

Seit Beginn der Diskussionen um Patientenverfügungen wurden Muster und Formulierungsvorschläge vorgestellt. Die gesicherten über 180 verschiedenen Entwürfe von Patientenverfügungen $^{24}$ geben Zeugnis einer breiten Meinungsvielfalt der Autoren oder Organisationen.

Patientenverfügungsmuster gehen unterschiedlichen methodischen Ansätzen nach und so existieren (1) Textbausteine, (2) Mustervorlagen mit einer vorgeprägten Meinung, (3) Ja/Nein Musterformulare, (4) Arbeitsvorlagen mit der Notwendigkeit der Individualisierung und (5) Informationsbroschüren mit Arbeitsvorlagen, die ausdrücklich zur Bearbeitung und persönlichen Anpassung auffordern ${ }^{25}$. Manchen Formularen sind Fallgeschichten beigefügt, die über medizinische Entscheidungskonflikte informieren.

Die Vielzahl von Patientenverfügungsmustern hat mehrfach die Frage aufgeworfen, ob es nicht eine bundeseinheitliche Musterpatientenverfügung geben könne. In einem Gutachten für das Bundesministerium der Gesundheit im Jahr 2002 kam eine interdisziplinäre Arbeitsgruppe zum Ergebnis, dass es »aufgrund der Vielzahl von individuellen Situationen und heterogenen weltanschaulichen Positionen« eine standardisierte allgemeingültige Patientenverfügung nicht geben $\mathrm{kann}^{26}$.

Bereits 1983 machte die amerikanische President's Commision, auf ein wesentliches Problem aufmerksam. Die vorhandenen »living wills« seien entweder armselig definiert und daher stark interpretationsbedürftig oder derart detailliert, dass sie eine »endlose Litanei von Zuständen « auflisteten, die am Lebensende nicht gewünscht würden. ${ }^{27}$ Die Schwierigkeit eine Patientenverfügung $\mathrm{zu}$ entwerfen, die prägnant, umfassend und ausreichend die Präferenzen von Patienten reflektiert, ist immer noch ungelöst. Je allgemeiner die Formulierung, desto offener für ungewünschte Interpretationen, je strikter und enger, desto größer die Gefahr, dass manchmal entgegen den tatsächlichen Wünschen der Patienten »am Buchstaben« geklebt wird.

Ein Ausweg aus diesem Dilemma besteht durch die eigene Festlegung der Verbindlichkeit der Patientenverfügung und die Bestimmung einer Person zur Auslegung der Patientenverfügung und Übertragung auf andere Entscheidungssituationen. Eine bewusste Entscheidung mittels Vollmacht für einen Stellvertreter, mit dem der Vollmachtgeber ausführlich gesprochen hat und welcher die Wünsche und Werte des Vollmachtgebers kennt, ist anzustreben.

24 Jacobi, T./May, A./Kielstein, R./Bienwald, W. (Hrsg.) (2004): Ratgeber Patientenverfügung. Vorgedacht oder selbstverfasst?

25 Jacobi, T./May, A./Kielstein, R./Bienwald, W. (Hrsg.) (2004): Ratgeber Patientenverfügung. Vorgedacht oder selbstverfasst?

26 Meran, J./Geißendörfer, S./May, A./Simon, A. (Hrsg.) (2002): Möglichkeiten einer standardisierten Patientenverfügung.

27 President's Commission for the Study of Ethical Problems in Medicine and Biomedical and Behavioral Research (1983), S. $136 \mathrm{ff}$. 


\section{Stellungnahmen, Berichte und deren Diskussion}

Im Jahr 2004 wurden einige Berichte und Stellungnahmen unterschiedlicher Gremien veröffentlicht. Den Auftakt machte im April die Bioethik Kommission des Landes Rheinland-Pfalz. Es folgte im Juni der Bericht der Arbeitsgruppe »Patientenautonomie am Lebensende « beim Bundesministerium der Justiz. Die interdisziplinäre Arbeitsgruppe wurde im September 2003 durch die Bundesministerin der Justiz eingesetzt, um Fragen der Verbindlichkeit von Patientenverfügungen zu klären und Eckpunkte für deren Abfassung zu erarbeiten und den damit verbundenen Gesetzgebungsbedarf darzustellen.

Darauf folgte Ende September die Stellungnahme der »Enquete-Kommission Ethik und Recht der modernen Medizin« des Deutschen Bundestages mit dem Zwischenbericht zu Patientenverfügungen vom 24.09.2004.

Der Nationale Ethikrat hat bisher keine Stellungnahme abgegeben. Es haben in 2004 zwei öffentliche Tagungen stattgefunden und eine Stellungnahme des Nationalen Ethikrats wurde bei der öffentlichen Tagung des Nationalen Ethikrats am 24.11.2004 in Münster zu »Selbstbestimmung am Lebensende « angekündigt ${ }^{28}$.

Im November 2004 hat das Bundesministerium der Justiz auf Grundlage der Empfehlungen der Arbeitsgruppe »Patientenautonomie am Lebensende « einen Referentenentwurf ${ }^{29}$ zur Stellungnahme an Fachkreise verschickt. Ein Gesetzesentwurf soll nach Planung des Bundesministeriums der Justiz im Frühjahr 2005 nach Einbringung in das Kabinett in das Gesetzgebungsverfahren eingebracht werden, sodass eine gesetzliche Regelung der Patientenverfügung zum 1.1.2006 in Kraft treten kann.

Im Folgenden werden die Ergebnisse der Arbeitsgruppe »Patientenautonomie am Lebensende « des Bundesjustizministeriums und der Zwischenbericht zu Patientenverfügungen der Enquete-Kommission »Ethik und Recht der modernen Medizin« in Ausschnitten vorgestellt und diskutiert.

Das Selbstbestimmungsrecht des Patienten ist ein hohes Gut, aber es vermag einen anderen Menschen nicht zu einer Handlung oder Unterlassung zu verpflichten, zu der er nicht bereit ist. Die Patientenverfügung hebelt somit die Gewissensfreiheit des behandelnden Arztes oder des beteiligten Behandlungsteams nicht aus. Nach dem Abschlussbereicht der Arbeitsgruppe »Patientenautonomie am Lebensende ${ }^{30}$ des Bundesministeriums der Justiz muss ein Arzt rechtzeitig für eine anderweitige Versorgung des Patienten sorgen, wenn er die Beendigung lebenserhaltender Maßnahmen mit seinem Gewissen nicht vereinbaren kann.

28 Das Wortprotokoll der Tagung wird auf der Internetseite des Nationalen Ethikrats http:// www.ethikrat.org veröffentlicht.

29 Entwurf eines 3. Gesetzes zur Änderung des Betreuungsrechts vom 1.11.2004, online abrufbar unter http://www.aem-online.de; siehe dazu die Pressemeldung vom 5.11.2004, online abrufbar unter: http://www.bmj.bund.de/media/archive/791.pdf.

30 Arbeitsgruppe »Patientenautonomie am Lebensende«, Abschlussbericht vom 10.06.2004, online abrufbar unter http://www.bmj.bund.de/media/archive/695.pdf. 
Der Abschlussbericht ${ }^{31}$ ist beginnend in Thesen abgefasst und enthält folgend Empfehlungen an den Gesetzgeber, wobei diese in die Themenkomplexe der Änderung des Betreuungsrechts (42-50) und der Änderung des Strafrechts (50-54) gegliedert sind. Die Arbeitsgruppe stellt dabei fest, dass aktive Sterbehilfe verboten bleiben muss. Aber zur Klarstellung der bereits jetzt schon geltenden Rechtspraxis soll der $\S 216$ StGB ergänzt werden.

Entwurf: »(3) Nicht strafbar ist

1. die Anwendung einer medizinisch angezeigten leidmindernden Maßnahme, die das Leben als nicht beabsichtigte Nebenwirkung verkürzt,

2. das Unterlassen oder Beenden einer lebenserhaltenden medizinischen Maßnahme, wenn dies dem Willen des Patienten entspricht.«

Dem Selbstbestimmungsrecht kommt zentrale Bedeutung zu. Das Recht zur Selbstbestimmung über den eigenen Körper gehört zum Kernbereich der durch das Grundgesetz geschützten Würde und Freiheit des Menschen. Für die Rechtmäßigkeit jedes ärztlichen Eingriffs am einwilligungsfähigen Kranken ist es erforderlich, dass die Patientin die Einwilligung sowohl für die Einleitung als auch für die Fortführung einer Therapie erteilen muss (6). Der Widerruf der Einwilligung ist jederzeit möglich. Die letzte Entscheidung trifft damit die einwilligungsfähige Patientin (7). Diese Entscheidung über die Vornahme oder die Fortsetzung einer lebenserhaltenden oder lebensverlängernden Behandlung ist auch dann möglich, wenn deren Beginn oder Fortsetzung ärztlich indiziert ist. Dies gilt nach Ansicht der gesamten Arbeitsgruppe ohne Rücksicht darauf, ob die Krankheit bereits einen unumkehrbar tödlichen Verlauf genommen hat und der Tod nahe bevorsteht.

Das zuvor wirksam ausgeübte Selbstbestimmungsrecht der Patientin bindet auch deren Vertreterin (Betreuerinnen, Bevollmächtigte). Die Vertreterin hat dem Patientenwillen Ausdruck und Geltung zu verschaffen (8).

Ohne Anhaltspunkte für ausgeübten Druck oder irrtümlich verfasste Patientenverfügungen muss nach dem Gesetzesentwurf in der vom Verfügenden gemeinten und nun eingetretenen Situation das Selbstbestimmungsrecht »in gleicher Weise wie bei einer aktuell getroffenen Entscheidung respektiert werden $\ll^{32}$. Dazu wird weiter festgestellt, dass der Mensch während seines gesamten Lebens Anspruch auf Achtung seiner Selbstbestimmung hat ${ }^{33}$. Die Prüfung der Patientenverfügung soll sorgfältig erfolgen, damit das »Leben geschützt und eine Heilbehandlung nicht deshalb unterlassen wird, weil eine Patientenverfügung falsch ausgelegt wird, eine nicht mehr aktuelle Patientenverfügung der Entscheidung zugrunde gelegt wird oder eine Willensäußerung beachtet wird, die nicht aus freien Stücken abgegeben worden ist. ${ }^{34}$

Bei vorliegender Willensbekundung, welche auf die konkrete Behandlungssituation zutrifft, ist eine Vertreterentscheidung nicht maßgeblich (10) und im Zweifel soll die

31 Die angegebenen Zahlen beziehen sich auf den Abschlussbericht vom 10.06.2004, wenn keine weitere Quelle angegeben ist.

32 Entwurf eines 3. Gesetzes zur Änderung des Betreuungsrechts vom 1.11.2004, S. 10.

33 Entwurf eines 3. Gesetzes zur Änderung des Betreuungsrechts vom 1.11.2004, S. 10.

34 Entwurf eines 3. Gesetzes zur Änderung des Betreuungsrechts vom 1.11.2004, S. 10. 
Vertreterin nach Beratung durch das Behandlungsteam entscheiden. Bei nicht eilbedürftigen Entscheidungen muss eine Betreuung angeregt werden, wenn keine Vertreterin bekannt oder zu ermitteln ist.

Ist der Wille der Patientin nicht bekannt und die Ermittlung des mutmaßliche Willens nicht möglich, so muss die medizinisch indizierte Behandlung mit dem Ziel der Erhaltung des Lebens begonnen werden (11).

Wenn zwischen Betreuerin und Ärztin und Behandlungsteam Konsens besteht zu dem erklärten oder mutmaßlichen Willen, muss bei der Verweigerung der Einwilligung der Betreuerin in die Einleitung oder Fortführung lebenserhaltender Maßnahmen das Vormundschaftsgericht nicht zur Genehmigung dieser konsentierten Feststellung beteiligt werden (11). Angehörige und Bezugspersonen sollen in den Entscheidungsfindungsprozess eingebunden werden, was regelmäßig bei einer Einzelfallberatung durch Klinische Ethik Komitees erfolgt ${ }^{35}$.

Die Kriterien zur Ermittlung der relevanten Daten und Informationen des mutmaßlichen Willens sind vielschichtig. Nach der Entscheidung des Bundesgerichtshofes von 1994 sind folgende Umstände zur Ermittlung des mutmaßlichen Willens heranzuziehen: »frühere mündliche oder schriftliche Äußerungen des Patienten, seine religiöse Überzeugung, seine sonstigen persönlichen Wertvorstellungen, seine altersbedingte Lebenserwartung oder das Erleiden von Schmerzen«.

Ergänzend können zur Ermittlung des Patientenwillens biographische Informationen herangezogen werden ${ }^{36}$. Dabei muss die generelle Frage der Relevanz von zuvor getätigten Äußerungen für die im zeitlichen Abstand liegende Therapieentscheidung geklärt werden. Wenn frühere Wünsche und Werte als Informationen zur Ermittlung des Patientenwillens herangezogen werden, so können Instrumente zur Vorbereitung einer Patientenverfügung wie Wertanamnese oder Werttagebuch aufschlussreich sein.

Die Methode der narrativen Wertanamnese fordert den Patienten auf, zu Krankheitsbildern, -situationen und Behandlungsalternativen Stellung zu beziehen ${ }^{37}$. Eine Wertanamnese kann auch als Vorbereitung zur Entscheidungs- und Selbstfindung beitragen, wenn die eigene Meinung zu einem Problemfeld noch nicht klar herausgebildet ist. Kielstein und Sass verstehen unter Wertanamnese die kritische Selbstanalyse und Selbstbewertung für die eigene Selbstverständigung aktuell oder prospektiv bei Ziel- und Entscheidungskonflikten. Die Interpretation von vorsorglichen Verfügungen ist ein Versuch des Verstehens und der Interaktion, denn es gilt, eine patientenorientierte Entscheidung im Fall der Einwilligungsunfähigkeit zu treffen.

Aufgrund der besonderen Nähe der gewillkürten Stellvertreterin (Bevollmächtigten) kann sie die eben beschriebenen Entscheidungen ohne Genehmigung des Vormundschaftsgerichts treffen (12). Die Möglichkeit zur Missbrauchskontrolle steht jederzeit zur Verfügung.

35 May, A. (2004): Ethische Entscheidungsfindung in der klinischen Praxis: Die Rolle des klinischen Ethikkomitees, Ethik in der Medizin, S. 242-252.

36 May, A. (2004): Ermittlung des Patientenwillens, in: Arbeitsgemeinschaft Rechtsanwälte im Medizinrecht (Hrsg.), Ärztliche Behandlung an der Grenze des Lebens, S. 59-78; Sass, H. M./ Kielstein, R. (2003): Patientenverfügung und Betreuungsvollmacht.

37 Sass, H. M./Kielstein, R. (2003): Patientenverfügung und Betreuungsvollmacht. 


\section{Wirksamkeitsvoraussetzungen und Validitätskriterien}

Die Arbeitsgruppe »Patientenautonomie am Lebensende« sieht Patientenverfügungen als formfreie Willensbekundungen, wobei die schriftliche Abfassung empfehlenswert ist (16).

Eine Reihe von Empfehlungen werden geäußert, z.B. Beratung durch Ärztin und/ oder fachkundige Organisationen; Überprüfung der inhaltlichen Konsistenz zu einer Organspendeerklärung. Der individuelle Gestaltungsspielraum soll jedoch nicht unnötig eingeschränkt werden. Daher spricht sich die Arbeitsgruppe auch für eine unbegrenzte zeitliche Wirksamkeit einer Verfügung aus.

Zur Auslegung einer Patientenverfügung sollten die zugrunde liegenden Motive und Wertvorstellungen dargestellt werden.

Die Arbeitsgruppe hat keine Musterpatientenverfügung erstellt, sondern Textbausteine mit einem breiten Meinungsspektrum vorgelegt (25-35), die in zwei unterschiedlichen Beispielsverfügungen veranschaulicht wurden (36-41).

Die vorgeschlagenen Textbausteine enthalten auch Vorschläge für die Reichweite einer Patientenverfügung, wenn die Entscheidungsfähigkeit nicht mehr vorhanden ist, und die Akzeptanz der medizinrechtlichen und ethisch gebotenen Selbstbestimmung des Patienten, welche eine möglichst umfassende Äußerungsmöglichkeit zur eigenen Behandlung gebietet. Diese Entscheidungen fallen im Einzelfall nicht leicht und Fragen zu »Sterben und Tod « sind oft noch Tabuthemen. Um dies zu ändern, muss es vermehrt Beratungsangebote geben. Auch ist eine individuelle Behandlung nur möglich, wenn bei Aufnahme in ein Altenheim, Pflegeheim, Krankenhaus oder zu Beginn einer ärztlichen Behandlung nach einer Patientenverfügung gefragt wird.

Das Erstellen einer Patientenverfügung setzt einen bewussten Prozess voraus und benötigt umfangreiche Überlegungen. Dabei müssen die nötigen Beratungsangebote ausgebaut und geschaffen werden. Einschränkungen der Entscheidungsmöglichkeiten, durch z.B. eine geforderte Schriftform oder eine erforderliche ärztliche Beratung, engen die Selbstbestimmung ein und müssen begründet werden. Regelungen der möglichen Missbrauchskontrolle sind ebenfalls Gegenstand der Empfehlungen der Arbeitsgruppe. Ein Weigerungsrecht, z.B. bei Bitten nach aktiver Sterbehilfe oder ärztlichen Maßnahmen, die mit dem Berufsethos des Arztes nicht vereinbar sind, findet sich im vorgestellten Gesetzesentwurf des BMJ.

Die Vorschläge der Arbeitsgruppe sind der Selbstbestimmung der Patientin verpflichtet und fördern die Gesundheitsmündigkeit.

Die Enquete-Kommission des Bundestages »Ethik und Recht der modernen Medizin « beschreibt in ihrem Zwischenbericht zu Patientenverfügungen die Notwendigkeit der »erforderlichen neuen Ausbalancierung der Prinzipien der Selbstbestimmung und der ärztlichen Fürsorgeverpflichtung $\ll^{38}$.

Im Ergebnis kommt die Mehrheit der Enquete-Kommission zur Empfehlungen der Einschränkung von Patientenverfügungen auf Fallkonstellationen, »in denen das

38 Enquete-Kommission »Ethik und Recht der modernen Medizin« des Bundestages (2004): Zwischenbericht Patientenverfügungen, Bundestagsdrucksache 15/3700, S. 8. 
Grundleiden irreversibel ist und trotz medizinischer Behandlung nach ärztlicher Erkenntnis zum Tode führen wird ${ }^{39}$. Diese Reichweitenbeschränkung wurde z.B. vom Vorsitzenden des Marburger Bundes Montgomery begrüßt und vom Präsidenten der Bundesärztekammer Hoppe abgelehnt ${ }^{40}$. In der von der Enquete-Kommission gelieferten Begründung wird ein möglicher »Druck auf ältere und/oder schwerkranke Menschen « und weitere Missbrauchsrisiken als Grund für die Reichweitenbeschränkung angeführt, die nach Ansicht der Enquete-Kommission eine »wohlbegründbare Einschränkung des Rechtes zur Selbstbindung $^{41}$ darstellen. Dabei soll der konkrete Mensch auch nicht mit einem Ideal der Selbstbestimmung »konfrontiert werden, das seinen Bedürfnissen nicht gerecht wird. Die sozialen und familiären Verflechtungen rechtfertigen für die Mehrheit der Enquete-Kommission den vorgeschlagenen Umgang mit Patientenverfügungen nach dem »Vorsichtsprinzip « ${ }^{42}$.

Es sind jedoch nun die Folgen der vorgeschlagenen Reichweitenbegrenzung zu prüfen und einige Argumente und Begründungen liefert die Enquete-Kommission selbst. Es gibt aber weitere Argumente:

Grundlegend für eine medizinische Behandlung ist deren Indikation. Aus der Indikation zu einer Untersuchung oder Behandlung folgt das Angebot zur Maßnahme. Zu diesem Therapievorschlag muss nun der aufgeklärte Patient entscheiden. Die Notwendigkeit zur Entscheidung des Patienten ruht auf zentralen rechtlichen und medizinethischen Grundwerten. Das Prinzip der Einwilligung nach Aufklärung bedeutet jedoch auch, dass die entscheidungsrelevanten Daten und Fakten einfühlsam mit dem Patienten besprochen werden müssen. Eine entsprechende Aufklärung darf nicht allein mit dem Aushändigen eines vorgefertigten Bogens erfolgen. Ein standardisierter Bogen und Informationsmaterial dürfen sehr wohl das Aufklärungsgespräch ergänzen, dieses aber nicht ersetzen. Das Gespräch mit dem behandelnden Arzt ist eine wichtige Facette des Entscheidungsfindungsprozesses, aber bei weitem nicht die einzige. Das therapeutische Angebot des behandelnden Arztes muss vom Patienten in den Kontext anderer Entscheidungsalternativen gestellt werden, was ein Recht auf Nichtbehandlung mit umfasst.

Nach den 2004 aktualisierten »Grundsätzen der Bundesärztekammer zur ärztlichen Sterbebegleitung « ist bei einwilligungsfähigen Patienten der aktuell geäußerte Wille des angemessen aufgeklärten Patienten $\gg z u$ beachten, selbst wenn sich dieser Wille nicht mit den aus ärztlicher Sicht gebotenen Diagnose- und Therapiemaßnahmen deckt. Das gilt auch für die Beendigung schon eingeleiteter lebenserhaltender Maßnahmen. Der Arzt soll Kranken, die eine notwendige Behandlung ablehnen, helfen, die Entscheidung zu überdenken.« Neu eingefügt in die Version der Grundsätze von

39 Enquete-Kommission »Ethik und Recht der modernen Medizin« des Bundestages (2004): Zwischenbericht Patientenverfügungen, Bundestagsdrucksache 15/3700, S. 38.

$40 »$ Die Ablehnung einer Behandlung ist bindend «, Gespräch von Hanno Kautz mit Jörg-Dietrich Hoppe, Ärztezeitung v. 09.11.2004.

41 Enquete-Kommission »Ethik und Recht der modernen Medizin« des Bundestages (2004): Zwischenbericht Patientenverfügungen, Bundestagsdrucksache 15/3700, S. 38.

42 Enquete-Kommission »Ethik und Recht der modernen Medizin« des Bundestages (2004): Zwischenbericht Patientenverfügungen, Bundestagsdrucksache 15/3700, S. 39. 
1998 ist auch die Formulierung »unter Beachtung des Selbstbestimmungsrechtes des Patienten«. Damit ist das Selbstbestimmungsrecht normativ verankert, was nur bestätigt, was bereits an anderen Stellen festgelegt ist.

Die Position der Enquete-Kommission stößt bei einer zweifelsfreien Patientenverfügung jenseits der beschränkten Reichweite auf Unverständnis, da in der einen Situation das Selbstbestimmungsrecht, mündlich geäußert, akzeptiert wird und in der anderen Situation der Festlegung durch eine Patientenverfügung nicht. Diese unterschiedliche Bewertung zweifelsfreier Äußerungen eines einwilligungsfähigen Patienten schränkt seinen Entscheidungsraum ein und aus der Wahlfreiheit eines einwilligungsfähigen, äußerungsfähigen Patienten wird ein verpflichtendes, nicht entscheidungsrelevantes Angebot. Diese Einschränkung der Entscheidungsbefugnis muss begründet werden, damit aus dem scheinbar lebenserhaltenden Angebot kein für das Individuum verpflichtendes, »unmoralisches Angebot« wird.

Wenn die Wahlfreiheit eingeschränkt wird, so kann dies auch auf einem entsprechenden Normalitätsbegriff beruhen. Was aber ist dann der bei Einschränkung der Reichweite einer Patientenverfügung zugrunde liegende Normalitätsbegriff? Es scheint zu sein, dass dann die Entscheidungsbefugnis hinter die medizinische Indikation einer Maßnahme zurücktritt. Kritiker haben bereits von einer »Zwangsbehandlung « gesprochen. Wenn die durch die Enquete-Kommission vorgeschlagene Regelung unreflektierte Entscheidungen von beeinflussten Menschen verhindern möchte, denen von außen eingeredet wird, dass ihr Leben nicht mehr lebenswert sei, so stellt sich die Frage, ob dazu die Reichweitenbegrenzung das geeignete Mittel ist. Unterstützende und sinnfindende Maßnahmen können schwerlich durch ein Gesetz zur Verfügung gestellt werden. Ein Schwerverletzter wird vom Notarzt auch bei vorliegender Patientenverfügung initial behandelt und bei ausreichender Zeit wird die Patientenverfügung betrachtet. Dabei ist die Patientenverfügung kein unumstößlichen, verpflichtende Diktum, sondern eine auf die aktuelle Situation anwendbare Entscheidung des Patienten.

Die damit zum Ausdruck gebrachte Meinung ist auf Grundlage der Wünsche und Werte des Patienten entstanden. Hierzu muss der Patient auch eine eigene Risikobewertung vornehmen. Das ist aber bei anderen lebensweltlichen Entscheidungen kaum anders. Bei Kreditinstituten wird bei Aktiengeschäften die Risikobereitschaft festgestellt. Nun ist die Reichweite von Aktiengeschäften denen im medizinischen Bereich nur begrenzt gleichzusetzen, aber für beide gilt das Risiko der Fehleinschätzung. Hier kehren wir nun an den Anfang der Überlegungen zur angemessenen, ausgewogenen neuen Ausbalancierung der Prinzipien der Selbstbestimmung und der ärztlichen Fürsorgeverpflichtung zurück und müssen uns nach der Verhältnismäßigkeit der Mittel fragen.

Für christlich orientierte Stimmen steht die besondere Präferenz für das Leben im Vordergrund und »die besondere Sorge für dessen Menschenwürdigkeit, die sich in Schutzwürdigkeit, aber auch in einer dem Lebensentwurf angemessenen Ausgestaltung entfaltet $\ll^{43}$.

43 Schardien, S. (2004): Menschenwürde und Sterbehilfe - wider einfache Vorgaben, in: Dabrock, P./Klinnert, L./Schardien, S. (Hrsg.), Menschenwürde und Lebensschutz. Herausforderungen theologischer Bioethik, S. 314 f. 
Einer unreflektierten Akzeptanz einer in ihren Auswirkungen nicht erfassten und unter Druck entstandenen Patientenverfügung muss entschiedenen entgegnet werden. Manche zukünftigen Situationen können in zeitlichem Abstand teilweise nicht in jeder Facette beurteilt werden und hierbei empfiehlt sich die eigene Festlegung des Verbindlichkeitsgrades der in der Patientenverfügung geäußerten Wünsche, was mit der Benennung einer Vertrauensperson in einer Vorsorgevollmacht kombiniert werden sollte.

Ein Schlüssel zu validen Patientenverfügungen wird in der Beratung im Vorfeld der Erstellung gesehen. Darin besteht Gemeinsamkeit in den Positionen der oben diskutierten Gremien, aber auch weiterer Stimmen in der Literatur.

Generell erfordert für die Enquete-Kommission »Selbstbestimmung notwendigerweise auch Information und Beratung ${ }^{44}$. Die Arbeitsgruppe beim BMJ ruft zur Dokumentation der Beratung durch Ärzte und »nichtärztliche, im Umgang mit Patientenverfügungen erfahrene Einrichtungen und Personen $\aleph^{45}$ auf. Weiter geht die Enquete-Kommission mit der »Förderung der Einrichtung und die Beauftragung unabhängiger multiprofessioneller Beratungsstellen ... oder alternativ die Bindung der Beratung an dafür qualifizierte Berater, die z.B. der Medizin, Pflege und Altenpflege angehören $\aleph^{46}$, aber auch Berater aus den »Bereichen Medizin, Rechtspflege, Psychologie, Pflege, Hospiz und Seelsorge ${ }^{47}$.

Bereits ein Gutachten für das Bundesministerium der Gesundheit hat 2001 die Bedeutung von Beratung und Beratungsstellen unterstrichen ${ }^{48}$. Konkretisiert wurden diese Überlegungen in der Erarbeitung eines Beratungskonzeptes für die Stadt Münster durch das Modellprojekt LIMITS ${ }^{49}$.

Als rechtspolitische Entscheidung muss beantwortet werden, welchen Stellenwert eine Beratung erhalten soll. Strengere Anforderungen an Information und Beratung zu Patientenverfügungen im Gegensatz zu aktuellen Entscheidungen im Gesundheitswesen sind begründungsbedürftig.

Erstmal ist nach Inhalten, Rahmenbedingungen der Beratung und Grundkompetenzen und sozialen Kompetenzen der Berater zu fragen. Inhaltlich ist auch nach den Inhalten von Informationen zu Patientenverfügungen zu fragen, bevor Standards der Beratung ${ }^{50}$ diskutiert werden. Ein Beratungsangebot kann sowohl von weltanschauli-

44 Enquete-Kommission »Ethik und Recht der modernen Medizin« des Bundestages (2004): Zwischenbericht Patientenverfügungen, Bundestagsdrucksache 15/3700, S. 9.

45 Arbeitsgruppe »Patientenautonomie am Lebensende«, Abschlussbericht vom 10.06.2004, S. 17.

46 Enquete-Kommission »Ethik und Recht der modernen Medizin« des Bundestages (2004): Zwischenbericht Patientenverfügungen, Bundestagsdrucksache 15/3700, S. 32.

47 Enquete-Kommission »Ethik und Recht der modernen Medizin« des Bundestages (2004): Zwischenbericht Patientenverfügungen, Bundestagsdrucksache 15/3700, S. 41.

48 Meran, J./Geißendörfer, S./May, A./Simon, A. (Hrsg.) (2002): Möglichkeiten einer standardisierten Patientenverfügung, S. 82-85.

49 Niewohner, $S$. (2004): Individuelle Beratung bei der Erstellung einer Patientenverfügung ein Konzept, in: Schulze, U./Niewohner, S. (Hrsg.): Selbstbestimmt in der letzten Lebensphase - zwischen Autonomie und Fürsorge, S. 194-206.

50 Vgl. Kress, H. (2004): Sterbehilfe. Geltung und Reichweite des Selbstbestimmungsrechts in ethischer und rechtspolitischer Sicht, S. 41. 
cher Offenheit geprägt sein als auch eine Empfehlung vor dem Hintergrund der geäuBerten Wünsche und Werte abgeben. Dies wird sich nach den Bedürfnissen des Ratsuchenden richten.

In einer Befragung von Ratsuchenden, denen Informationsmaterial zu Patientenverfügungen und die »Bochumer Patientenverfügung « von Sass/Kielstein geschickt wurde, gaben $71 \%$ der Antwortenden die erfolgte Beratung zu Patientenverfügungen $a^{51}$. Einen Einfluss durch die Beratung mit Arzt, Familie, Bekannten/Freunden, Jurist bzw. Betreuungsverein gab ein Großteil der Befragten an, wobei die Gespräche mit Seelsorger und Gericht überwiegend keine Beeinflussung darstellten $^{52}$. Am häufigsten wurde der Wunsch nach einer Beratung durch den Hausarzt geäußert, gefolgt von Freunden, Familie und Juristen ${ }^{53}$.

Die Vorstellungen von einer entsprechenden Beratung differieren. So ist, unabhängig von einer Verpflichtung zur Beratung, nach den Inhalten einer solchen Beratung zu fragen. Bei der Entwicklung von Standards und Rahmenbedingungen von Beratung zu Patientenverfügungen sollten Erfahrungen mit der humangenetischen Beratung und anderen Mediengebieten berücksichtigt werden.

Die aktuell geforderte Beratungspflicht bei Patientenverfügungen, die über die von der Mehrheit der Enquete-Kommission des Bundestags beschriebene Reichweite hinausgehen, erfordert weitere Diskussionen.

Kress sieht in der Entwicklung von Verfahren, Kriterien und Qualitätsstandards für Beratung zu Patientenverfügungen, die sich am »Individuum und seinen Freiheitsrechten $«$ orientieren, eine medizin- und sozialethische Frage ${ }^{54}$.

Nach dem Prozess des Entstehens einer Patientenverfügung stellt sich die Frage nach dem Umgang und der Auslegung einer Patientenverfügung durch den Entscheidungsträger. Dazu kann auch zur Beratung des Entscheidungsträgers die Beteiligung eines Ethik Komitees sinnvoll sein.

\section{Rolle des Klinischen Ethik Komitees und der Vormundschaftsgerichte bei der Ent- scheidungsfindung und-überprüfung}

Das Selbstbestimmungsrecht ist nicht nur für die Arbeitsgruppe »Patientenautonomie am Lebensende « von großer Bedeutung. Für die Rechtmäßigkeit jedes ärztlichen Eingriffs am einwilligungsfähigen Kranken ist es erforderlich, dass die Patientin oder der Patient die Einwilligung sowohl für die Einleitung als auch für die Fortführung einer Therapie erteilen muss. Der Widerruf der Einwilligung ist jederzeit möglich. Die letzte Entscheidung trifft damit die einwilligungsfähige Patientin oder der einwilli-

51 May, A./Brandenburg, B. (2004): Einstellungen medizinischer Laien zu Behandlungsverfügungen, S. 12.

52 May, A./Brandenburg, B. (2004): Einstellungen medizinischer Laien zu Behandlungsverfügungen, S. 12.

53 May, A./Brandenburg, B. (2004): Einstellungen medizinischer Laien zu Behandlungsverfügungen, S. 12.

54 Vgl. Kress, H. (2004): Sterbehilfe. Geltung und Reichweite des Selbstbestimmungsrechts in ethischer und rechtspolitischer Sicht, S. 41. 
gungsfähige Patient. Bei Aufnahme in ein Altenheim, Pflegeheim, Krankenhaus oder zu Beginn einer ärztlichen Behandlung soll nach einer Patientenverfügung gefragt werden.

Entscheidend für Patientenverfügungen als Instrument der Kommunikation moralisch Fremder ist die Detailliertheit und auch der Grad der Informiertheit. Die Erstellung einer Patientenverfügung ist eine längere Aufgabe und darf nicht unreflektiert durch die Unterschrift unter ein beliebiges Formular erfolgen. Patientenverfügungen sind ein Gestaltungsinstrument als Ausdruck der Selbstbestimmung des Patienten, dem jedoch einzelne Grenzen gesetzt sind.

Die Enquete-Kommission »Ethik und Recht der modernen Medizin« des Bundestages schlägt zur Kommunikation zwischen den Beteiligten und als prozedurales Erfordernis ein Konsil aus behandelndem Arzt, rechtlichem Vertreter, einem Mitglied des Pflegeteams und einem Angehörigen vor ${ }^{55}$. Dabei muss noch geklärt werden, welche Bereiche nun durch das Konsil im Rahmen einer Entscheidung (Feststellung) und was als Beratung durch das Konsil bearbeitet werden soll. Die unterschiedlichen Implikationen und Modelle sollen nachfolgend verdeutlicht werden.

In den USA wurden Klinische Ethik Komitees zunächst durch konfessionelle Träger von Krankenhäusern in den fünfziger Jahren des vergangenen Jahrhunderts zusammengesetzt, um sich der Frage nach dem eigenen Profil und nach religiös geprägten Moralauffassungen in einer immer offensiver wertepluralistischen Kultur einer liberalen Gesellschaft zu stellen. Die Catholic Hospital Organization empfahl bereits 1949 die Einrichtung von Gremien der Ethikberatung. Damit versuchten kirchliche Träger eine Antwort auf die Herausforderung, wie in der immer offensiver wertepluralistischen Kultur einer liberalen Gesellschaft überhaupt noch religiös geprägte Moralauffassungen zur Geltung gebracht werden können. Auch sollten sie die »Erweiterung medizinischer Handlungsmöglichkeiten « reflektieren ${ }^{56}$.

Die nächste Phase der Entwicklung Klinischer Ethik Komitees wird durch Probleme der Verteilungsgerechtigkeit von knappen Behandlungsmitteln, vor allem von begehrter HighTech-Medizin z.B. im Fall der Hämodialyse, vorangetrieben. Dabei trifft das Klinische Ethik Komitee eine Entscheidung auf Basis einer nicht völlig transparenten Zusammensetzung. Instruktiv ist hierzu die bildliche Darstellung des Seattle Dialysis Committee im LIFE Magazin, wo vor einer hell erleuchteten Bücherwand die Mitglieder des Komitees abgebildet sind, ohne die Gesichter erkennen zu lassen. Der Untertitel »They Decide Who Lives, Who Dies« vermittelt die exponierte Rolle der Komiteemitglieder $^{57}$.

Durch den Rechtsstreit zur Behandlung von Karen Ann Quinlan und die Empfehlung des Gerichts zur Einschaltung eines Ethik Komitees schlossen sich andere Gerichte dieser Empfehlung an ${ }^{58}$. In Folge um den Streit über die Autonomie wurde

55 Enquete-Kommission »Ethik und Recht der modernen Medizin« des Bundestages (2004): Zwischenbericht Patientenverfügungen, Bundestagsdrucksache 15/3700, S. 43.

56 Steinkamp, N./Gordijn, B. (2003): Ethik in der Klinik, S. 95.

57 Alexander, S. (1962): They decide who lives, who dies. Life, 9/11/1962.

58 Jonsen, A. (1998): The Birth of Bioethics, S. 363. 
durch den President's Commission Report (1983) zu Fragen des Behandlungsabbruchs ein Modellentwurf für die Satzung zukünftig einzurichtender Ethik Komitees bekannt gemacht. Mit dem Prinzip der Interdisziplinarität betont der Report die Notwendigkeit verschiedener Sichtweisen zur Diskussion einer Fragestellung. Eng damit verbunden ist die Vorstellung der Repräsentativität der in den Behandlungsprozess eingebundenen Gruppen im Krankenhaus.

In Deutschland hat die gemeinsame Empfehlung der Evangelischen und der Katholischen Krankenhausverbände 1997 einen richtungweisenden Impuls auf die Entwicklung der institutionalisierten Ethikberatung in Deutschland ausgeübt ${ }^{59}$. Nach dieser Empfehlung ist das Ethik Komitee »in einem Krankenhaus angesiedelt, das ethische Konflikte kennt und bewusst angehen will«. Zu diesem Ziel sollen Einzelfälle besprochen und begutachtet werden, um »nach bestem Wissen und Gewissen im gemeinsamen Diskurs die relativ beste Lösung zu finden ${ }^{60}$.

Das Ethik Komitee soll interdisziplinär und ausgewogen mit Vertretern des ärztlichen, pflegerischen Bereichs, des Verwaltungsbereichs und des Sozialdienstes besetzt sein. Als externe Mitglieder sollen ein Bürger mit »gesundem Menschenverstand « und christlicher Grundhaltung, ein Jurist und ein Seelsorger im Komitee vertreten sein. Die Voten des Ethik Komitees sollen »das Urteil der Entscheidungsträger wohl erhellen, aber nicht ersetzen«. Für die konfessionellen Krankenhausverbände entspricht die Einführung von Ethik Komitees »dem Bedürfnis eines christlichen Menschenbildes « ${ }^{61}$.

Bereits vor der Empfehlung der konfessionellen Krankenhausverbände wurden vereinzelt »Consensusgespräche über die weitere Therapie « durchgeführt ${ }^{62}$ oder Konsildienste durch Ethikberater eingerichtet. Weiteren Anlass zur Einrichtung von Ethik Komitees erhalten Krankenhäuser durch die Zertifizierung, z.B. durch die Kooperation für Transparenz und Qualität im Krankenhaus (KTQ): »1. Durch welche organisatorischen Maßnahmen ist die Berücksichtigung ethischer Problemstellungen im Krankenhaus gewährleistet (z.B. durch die Einrichtung eines Ethikkomitees im Krankenhaus oder anderer Gruppen mit der gleichen Zielsetzung, ggf. unter Einbeziehung der Krankenhausseelsorge)? (Frage 5.4.1, Version 4, 2002). Gremien der Ethikberatung und damit Ethik Komitees sollen nach Beschluss der Gesundheitsministerkonferenz vom 20./21.06.2002 im Bereich der Sterbebegleitung eine größere Bedeutung erhalten.

Klinische Ethik-Komitees bieten das Forum zur Diskussion unterschiedlicher Moralurteile und unterschiedlicher Intuitionen. In Moralurteilen werden Einstellungen

59 Deutscher Evangelischer Krankenhausverband, Deutscher Katholischer Krankenhausverband (1997): Ethik-Komitee im Krankenhaus.

60 Deutscher Evangelischer Krankenhausverband, Deutscher Katholischer Krankenhausverband (1997): Ethik-Komitee im Krankenhaus, S. 13.

61 Deutscher Evangelischer Krankenhausverband, Deutscher Katholischer Krankenhausverband (1997): Ethik-Komitee im Krankenhaus, S. 21.

62 Zumtobel, V./Finke, U.(1994): Universitätsklinik St.-Josef-Hospital Bochum, Hals-NasenOhren-Klinik im St.-Elisabeth-Hospital, in: Medizinische Fakultät der Ruhr-Universität Bochum (Hrsg.), Festschrift zum fünfundzwanzigjährigen Bestehen der Medizinischen Fakultät der Ruhr-Universität Bochum, S. 98. 
zu akuten Situationen zum Ausdruck gebracht. Die Institution Krankenhaus kann die Diskussion dieser unterschiedlichen Moralurteile fördern und ein interdisziplinäres Forum bieten. Dadurch wird die Entscheidungsbefugnis des Patienten oder seines Stellvertreters (Bevollmächtigter oder rechtlicher Betreuer) nicht verlagert, sondern eine Beratung des Entscheidungsträgers erreicht. In der Diskussion der Entscheidungsgrundlage können die Beweggründe des Entscheidungsträgers dargestellt werden, was zur Akzeptanzförderung bei anderen Akteuren beiträgt. Bei Entscheidungen innerhalb der Organisation Krankenhaus werden diese transparenter und innerhalb des Gestaltungsspielraums nachvollziehbarer. Die Präsenz eines Ethik Komitees in der Klinik erhöht die Arbeitszufriedenheit, da die Partizipation die Möglichkeiten zur Bewältigung von »moralischem Stress « verbessert.

In der Erklärung der konfessionellen Krankenhausverbänden findet sich zum Auftrag Klinischer Ethik Komitees: »Probleme im Krankenhaus entstehen dann, wenn die allgemeinen Grundsätze ärztlichen und pflegerischen Handelns entweder zu unspezifisch sind, als dass man daraus eine konkrete Handlungsstrategie ableiten könnte, oder wenn sie miteinander kollidieren. (...) Ethik-Komitees werden sich in solchen Fällen bemühen, nach bestem Wissen und Gewissen im gemeinsamen Diskurs die relativ beste Lösung zu finden. Entscheidungen »ohne Rest« wird es hier oft nicht geben. Christliche Leitlinie des Handelns wird die aus der Liebe Gottes gespeiste Liebe zum Nächsten und zugleich das Bewusstsein der eigenen Vergebungsbedürftigkeit sein. Das schützt vor einer bequemen Selbstgenügsamkeit, die es bei mangelnder Klarheit vorzieht, die eigene und zufällige Meinung aufzudrängen, statt nach einer Lösung zu suchen, die mindestens im Komitee und im Kreis der Beteiligten kommunikabel und konsensfähig ist. $\ll^{63}$. In diesem Sinne ist die Einrichtung Klinischer Ethik Komitees ein aktiver Beitrag zur Diskussion über Selbstverständnis und Qualität der Patientenversorgung. Insbesondere fragen sich viele konfessionelle Krankenhäuser nach dem »Proprium « ihrer Arbeit und einen Eindruck zu möglichen Antworten liefert die obige Beschreibung, die je nach Kontext spezifiziert und konkretisiert werden muss.

Als Ideal der Arbeit eines Klinischen Ethik Komitees (KEK) stellt es beratend »ein Forum für schwierige und kontroverse moralische Entscheidungen bereit. ${ }^{64 \cdot} \mathrm{Zu}$ den Aufgaben eines Klinischen Ethik Komitees gehören (1) die Weiterbildung in medizinischer Ethik, (2) einzelfallbezogene Beratung, entweder im Rückblick oder bezogen auf aktuelle Behandlungsentscheidungen, die die Betroffenen als moralisch problematisch wahrnehmen und (3) die Entwicklung von Leitlinien, welche die moralische Integrität der Institution gewährleisten sollen.

Zur Arbeit Klinischer Ethik Komitees müssen organisationsspezifisch Regeln der Institutionalisierung, der Aktivierung, Zuständigkeit und der Deliberation und der

63 Deutscher Evangelischer Krankenhausverband, Deutscher Katholischer Krankenhausverband (1997): Ethik-Komitee im Krankenhaus, S. 13 f.

64 Klinisches Ethik Komitee der Medizinischen Hochschule Hannover (MHH) (2001): Präambel, in: Kettner, M./May, A. (Hrsg.) Ethik-Komitees in der Klinik. Zur Moral einer neuen Institution, in: Rüsen, J.: Jahrbuch des Kulturwissenschaftlichen Instituts im Wissenschaftszentrum NRW 2000/2001, S. 492. 
Darbietung und des Umgangs mit Beratungsergebnissen aufgestellt werden ${ }^{65}$. Die ausgeformten Regeln bilden das Fundament und ein Gerüst für die Arbeit eines Klinischen Ethik Komitees mit den eben genannten Funktionsbeschreibungen.

Das vormundschaftsgerichtliche Genehmigungsverfahren überprüft die beabsichtigte Entscheidung des Betreuers oder Bevollmächtigten. Für Knieper sind Vormundschaftsrichter zur Willensermittlung des Betreuten besonders geeignet: »Vormundschaftsrichter sind spezialisiert auf die Willensermittlung von Personen, die aufgrund einer Krankheit oder Behinderung ihre eigenen Angelegenheiten nicht mehr allein regeln können (§ 1896 I BGB); [denn] sie sind aufgrund ihrer alltäglichen Arbeit in der Lage, die einzuholenden medizinischen Gutachten zu verstehen $\ll^{66}$. Ungeachtet der eindeutigen Rechtslage bewerten Vormundschaftsrichter die Beendigung der Nahrungszufuhr über eine Sonde zu 31,9\% als aktive Sterbehilfe, wobei der Verzicht auf Nahrungszufuhr über eine Sonde zu $8,4 \%$ als aktive Sterbehilfe eingestuft wird ${ }^{67}$.

Vormundschaftsrichter können bei Bedarf zusätzlich zu einer Patientenverfügung Zeugen zum Herausfinden des Willens des Patienten unter Eid nehmen und verfügen für Bauer über weiterreichende Möglichkeiten der Willensermittlung als Ärzte oder Klinische Ethik Komitees ${ }^{68}$. Bei Einigkeit über die Auslegung einer Patientenverfügung durch Arzt und Betreuer sieht Kutzer bei einer Regelkontrolle durch das Vormundschaftsgericht ein $\gg$ überzogenes >staatspaternalistisches $<$ Misstrauen gegen die Entscheidungsfähigkeit von Arzt, Betreuer und Betroffenen « und der Vormundschaftsrichter darf nicht zum Schicksalsbeamten werden ${ }^{69}$.

Die Ermittlung des mutmaßlichen Willens erfolgt indirekt durch Befragungen, wenn keine Behandlungswünsche dokumentiert wurden. Die indirekte Ermittlung der Wünsche und Werte scheint für Hartmann mit einer wesentlich größeren Unsicherheit behaftet als der vorab (mündlich oder schriftlich) geäußerte Wille, da zur Ermittlung des mutmaßlichen Willens »regelmäßig viele äußere Aspekte herangezogen werden, die den Betroffenen tangieren, letztlich aber nicht aus ihm heraus resultieren ${ }^{70}$, und damit durch verschiedene Informationsquellen und Interpretationen immer mit der Gefahr verbunden sind, den mutmaßlichen Patientenwillen zu verfehlen, wenn frühere

65 May, A./Kettner, M. (2002): Beratung bei der Entscheidung zum Therapieabbruch durch Ethik-Konsil oder Klinisches Ethik-Komitee, in: May, A./Geissendörfer, S./Simon, A./ Strätling, M. (Hrsg.), Passive Sterbehilfe: besteht gesetzlicher Regelungsbedarf?, S. 188192.

66 Knieper, J., Vormundschaftsgerichtliche Genehmigung des Abbruchs lebenserhaltender Maßnahmen, NJW 1998, S. $2720 \mathrm{f}$.

67 Simon, A./Lipp, V./Tietze, A./Nickel, N./van Oorschot, B., Einstellungen deutscher Vormundschaftsrichterinnen und -richter zu medizinischen Entscheidungen und Maßnahmen am Lebensende: erste Ergebnisse einer bundesweiten Befragung, Medizinrecht 2004, S. 303-307.

68 Bauer, A., Juristische Argumentationslinien und die Funktion der Justiz im Rahmen der Sterbehilfedebatte, Betreuungsrechtliche Praxis 2002, S. 60-62.

69 Kutzer, K., Der Vormundschaftsrichter als »Schicksalsbeamter«? Zeitschrift für Rechtspolitik 2003, S. 213-216.

70 Hartmann, T., Patientenverfügung und Psychiatrische Verfügung - Verbindlichkeit für den Arzt, NStZ 2000, S. 113-115. 
Äußerungen und Informationen auf die jetzige Situation extrapoliert ${ }^{71}$ werden. Taupitz sieht die Gefahr, dass durch die Wertung und Interpretation früherer Äußerungen im Rahmen der Ermittlung des mutmaßlichen Willens der eigene Wille des zukünftigen Entscheiders als der Wille des Patienten ausgegeben wird ${ }^{72}$.

Neben den in der Entscheidung BGH Kempten genannten Kriterien zur Ermittlung des mutmaßlichem Willens wird »der Bezug auf Äußerungen, die in einem zeitlich überschaubaren und inhaltlich eindeutigen Zusammenhang zur Sterbesituation stehen, der Bezug auf Äußerungen, die in einem ernsthaften Dialog mit dem Betroffenen gefallen sind, sowie eine verbindliche Dokumentation darüber, wer auf Grund welcher Äußerungen zu welchen Schlussfolgerungen gekommen ist « ${ }^{73}$, vorgeschlagen. Diese Trennung von medizinisch-wissenschaftlichen und medizinethischen Befunden nimmt der »Bochumer Arbeitsbogen zur medizinethischen Praxis ${ }^{74}$ vor und bietet sich als Instrument zur ethischen Fallanalyse an.

Wagenitz und Engers merken an, dass sich der Richter mit einer ethischen Entscheidung alleine finde, wenn die gerichtliche Willenserforschung des Patienten kein klares Bild ergibt, und fragen sich, »ob eine solche Entscheidung beim Vormundschaftsgericht in besseren Händen ist als bei Ärzten, Angehörigen oder einem gar vom Patienten selbst vorgeschlagenen oder bevollmächtigten Vertreter. Unstreitig schafft ein justizielles Verfahren für alle Beteiligten Sicherheit, aber es verlängert, wie das Beispiel (OLG Frankfurt am Main 15.07.1998) zeigt, unter Umständen menschliches Leiden erheblich $\ll^{75}$. Der Bundesgerichtshof hat mit seiner Entscheidung vom 17.03.2003 bedauerlicherweise keine zeitlichen Vorgaben für ein vormundschaftsgerichtliches Verfahren gemacht, sodass der Patient zweieinhalb Monate nach der Entscheidung des BGH verstarb, ohne dass es eine vormundschaftsgerichtliche Entscheidung des zuständigen Amtsgerichts gegeben hätte.

Vorgeschlagen wird nun bei Entscheidungen am Lebensende die Beteiligung von Ethik-Konsilen ${ }^{76}$ und mit Blick auf die USA Ethik-Komitees, wobei nach Deutsch Ethik Komitees aus Ärzten, Pflegenden, Ethikern und Familienangehörigen bestehen ${ }^{77}$.

Bei Interpretationsschwierigkeiten des mutmaßlichen Willens können Klinische Ethik Komitees eine Hilfestellung für den Entscheidungsträger sein, indem die Argumente aus verschiedenen Blickwinkeln und Perspektiven analysiert werden ${ }^{78}$. Dazu ist die Frage nach dem legitimierten Entscheidungsträger der Ausgangspunkt für die

71 Taupitz, J., Die Macht der Gesetze ist beschränkt, Deutsches Ärzteblatt 2001, S. 2941.

72 Taupitz, J., Die Macht der Gesetze ist beschränkt, Deutsches Ärzteblatt 2001, S. 2941.

73 Wunder, M., Sterbebegleitung für nicht Sterbende, Mabuse 1998, Nr. 116, 28.

74 Viefhues, H./Sass, H. M. (1987): Der Bochumer Arbeitsbogen zur medizinethischen Praxis.

75 Wagenitz, T./Engers, M., Anmerkung zu OLG Frankfurt - BGB $\S 1904$, FamRZ 1998, S. $1256 \mathrm{f}$.

76 Klie, T., Gesetzliches Vertretungsrecht für Angehörige, Betreuungsrechtliche Praxis 2002, S. 91-96; Klie, T., Sterben in Würde - zwischen Autonomie und Fürsorge, Z. f. Gerontol. Geriat. 2003, S. 347-354.

77 Deutsch, E., Verfassungszivilrecht bei der Sterbehilfe, NJW 2003, S. 1567 f.

78 Vgl. May, A./Kettner, M. (2002): Beratung bei der Entscheidung zum Therapieabbruch durch Ethik-Konsil oder Klinisches Ethik-Komitee, in: May, A./Geissendörfer, S./Simon, A./Strätling, M. (Hrsg.), Passive Sterbehilfe: besteht gesetzlicher Regelungsbedarf?, S. 188-192. 
Überlegungen des Klinischen Ethik Komitees. In einer Beratung eines Ethik Komitees werden Moralurteile gewichtet und auf den Einzelfall bezogen. Die Hermeneutik der direkten Äußerungen und der indirekt ermittelten Einstellungen kann durch das Klinische Ethik Komitee differenziert und aus verschiedenen interprofessionellen und multidisziplinären Perspektiven erfolgen. Konflikte können durch verschiedene Positionen zu »balancierten Konflikten« werden ${ }^{79}$.

Nach der Präambel der Satzung des Klinischen Ethik Komitees der Medizinischen Hochschule Hannover (MHH) soll das Komitee »einen Beitrag zur Kultur der MHH und zum Klima und Stil in der Patientenversorgung leisten« und »Verantwortung, Selbstbestimmungsrecht, Vertrauen, Respekt, Rücksicht und Mitgefühl« sollen als gelebte moralische Werte die Entscheidungen und den Umgang an der MHH prägen. Der Bezug auf die Vier Prinzipien nach Beauchamp und Childress (Respekt vor der Selbstbestimmung des Patienten; Nichtschadensgebot; Gutes tun/Fürsorge; Gerechtigkeit) scheint eine breite Basis für die Arbeit eines Ethik Komitees zu haben ${ }^{80}$. Die konzeptionellen Überschneidungen zu kasuistischen Ansätzen müssen an anderer Stelle untersucht werden. Die Frage nach der Ethik eines Klinischen Ethik Komitees oder allgemeiner eines ethischen Beratungsgremiums soll an dieser Stelle benannt werden, ohne ein abgeschlossenes Theoriekonzept darstellen zu können.

Die Gesprächsbedingungen innerhalb der Beratung des Klinischen Ethik Komitees durch eine konstruktive, die Gleichheit der Gesprächspartner achtende Auseinandersetzung müssen in hierarchisch geführten Organisationen wie einem Krankenhaus besondere Beachtung finden. Dies ist besondere Aufgabe des Moderators oder Vorsitzenden des Klinischen Ethik Komitees. Weiterhin ist zur Funktion eines ethischen Beratungsgremiums seine Besetzung vorentscheidend. Die konfessionellen Krankenhausverbände fordern vom Vorsitzenden des Ethik Komitees professionelle Moderationskompetenzen und von allen Komiteemitgliedern die Kenntnis unterschiedlicher Argumentationsfiguren, welche »kontrovers aber fair « innerhalb kurzer Zeit gegeneinander abgewogen werden können. Dabei ist es gewollt, dass die Komiteemitglieder ihren eigenen Standpunkt »im Spiegel der Standpunkte« anderer Teilnehmer konturieren.

Die Zusammensetzung von Ethik-Konsilen ist unterschiedlich organisiert und es lassen sich neben der vorteilhaften Flexibilität jedoch Fragen nach dem Einfluss der situativen Zusammensetzung stellen. Der beim Ethik-Konsil anwesende Ethiker muss unterschiedliche Perspektiven einnehmen, wenn eine Perspektivenvielfalt als anregend für einen diskursiven Prozess angesehen wird. Instruktiv beschreibt Engelhardt einen Ethik-Berater als (1) juristischen Ratgeber, (2) Risiko Manager, (3) Konfliktschlichter, Mediator, (4) Informationsquelle und (5) Ethiker ${ }^{81}$.

Eine konkrete Ethikberatung im Einzelfall kann eine Optimierung des Bewertungsprozesses der medizinisch beabsichtigten Alternative leisten und dem Entschei-

79 Müller-Busch, C. (2002) Zur Behandlung von ethischen Problemen und Konflikten in der Palliativmedizin, Zeitschrift für Palliativmedizin, S. 71.

80 Engelhardt, H. T., Healthcare ethics committees: re-examining their social and moral functions, HEC Forum 1999, S. 87-100.

81 Engelhardt, H. T., The bioethics consultant: Giving moral advice in the midst of moral controversy, HEC Forum 2003, S. 362-382. 
dungsträger wegen der Reichweite der Entscheidung bei der Abschätzung aller Handlungsfolgen helfen und ihn unterstützen. Dabei muss klar bleiben, dass der Vertreter der Entscheidungsträger ist und bleibt. Dieser kann durch das Ethik Komitee eine Unterstützung erfahren, aber die Verantwortung für die Entscheidung darf nicht an eine Gruppe delegiert werden. Eine Ethikberatung kann auch klärend für moralische Probleme weiterer Beteiligter wirken. Die vorgeschlagene prozedurale zwingende Beteiligung von Ethik-Konsilen oder Ethik Komitees wirft eine Reihe von praktischen Fragen auf.

\section{Schlussbemerkung}

Die Sicherung und der Schutz der Selbstbestimmung des Einzelnen muss zentraler Gegenstand der gesellschaftlichen Diskussion sein. Dabei sind unterstützende Angebote bei der Information, Beratung und der Erstellung von Patientenverfügungen zu betrachten. Patientenverfügungen sind schon längst kein gesellschaftliches Randphänomen mehr, welches durch Vertrauen in »gute Ärzte « verschwinden wird. Unterschiedliche Wertsysteme werden $\mathrm{zu}$ differenzierten Behandlungswünschen und Therapieangeboten führen. Diese persönlichen Entscheidungen beruhen auf moralischen Überzeugungen. Unterschiedliche Überzeugungen können durch ethische Fallbesprechungen und/oder Ethik-Komitees diskursiv in ein Gespräch gebracht werden und dies nicht allein in Krankenhäusern, sondern auch in der stationären und ambulanten Altenpflege.

Bei der Erstellung einer Patientenverfügung spielt Beratung eine große Rolle, diese sollte aber nicht verpflichtend sein im Sinne eines Wirksamkeitserfordernisses. Jeder einwilligungsfähige Mensch hat das Recht auf unvernünftige Entscheidungen. Es benötigt unterstützende »fürsorgliche« Angebote, welche durch z.B. Beratung die Gesundheitsmündigkeit fördern.

Möglicherweise wird der vorliegende Referentenentwurf von einzelnen Diskussionsteilnehmern je nach ihren persönlichen Überzeugungen als unmoralisch angesehen, aber unethisch ist der Entwurf sicher nicht. Der Stellenwert von Selbstbestimmung und Autonomie muss ausbalanciert werden, damit Menschen ihre medizinische Versorgung entscheiden und beeinflussen können, ohne eine Zwangsbehandlung fürchten zu müssen. Ein praxisnahes handhabbares Konzept ethischer Prinzipien bieten die Vier Prinzipien (Respekt vor der Selbstbestimmung des Patienten, Nichtschadensgebot, Wohltätigkeit/Fürsorge und Gerechtigkeit) nach Beauchamp/Childress.

Es muss auch erlaubt sein, den Festlegungen einer Patientenverfügung in einer begründeten Einzelfallentscheidung nicht folgen zu müssen. Eingriffe in das Selbstbestimmungsrecht des Patienten müssen begründet werden. Gesellschaftliche Festlegungen müssen dem Anspruch der Patientenrechte und der Gesundheitsmündigkeit folgen und vielfältige Beratungs- und Unterstützungsmöglichkeiten zur Verfügung stellen.

Persönliche Intuitionen und Überzeugungen zu würdevollem Leben und Sterben differieren bereits heute, was auf unterschiedlichen Ansichten zu medizinischen Handlungsoptionen und technischen Möglichkeiten beruht. Einen gesellschaftlichen Konsens darüber herzustellen, scheint wenig aussichtsreich. Die gesellschaftliche 
Festlegung auf den Lebensschutz des Einzelnen kommt durch das Prinzip »in dubio pro vita« zum Ausdruck und vormundschaftsgerichtliche Prüfungsverfahren sind Ausdruck dieser rechtsstaatlichen Garantien. Sollten Zweifel an den Patientenwünschen nicht erkennbar sein, so sind diese Überzeugungen des Patienten Entscheidungsgrundlage, so unvernünftig diese Wünsche nach den eigenen Wertesystemen erscheinen mögen. Die Gesellschaft hat die Pflicht zur Sicherung der Selbstbestimmung des Einzelnen auch bei $»$ langfristigen Dispositionen $\ll^{82}$.

82 Gerhardt, V. (2004): Selbstbestimmung in der Biopolitik, Vortrag bei der öffentlichen Tagung des Nationalen Ethikrats am 24.11.2004 in Münster zu »Selbstbestimmung am Lebensende $\ll$. 